Théologiques

Théologiques

\title{
L'attaque religieuse contre la science : une bataille pour la définition de la vérité et de la connaissance, ou de la politique?
}

\section{Harvey B. Sarles}

Volume 2, numéro 1, mars 1994

Création et créativité

URI : https://id.erudit.org/iderudit/602399ar

DOI : https://doi.org/10.7202/602399ar

Aller au sommaire du numéro

\section{Éditeur(s)}

Faculté de théologie de l'Université de Montréal

ISSN

1188-7109 (imprimé)

1492-1413 (numérique)

Découvrir la revue

Citer cet article

Sarles, H. B. (1994). L'attaque religieuse contre la science : une bataille pour la définition de la vérité et de la connaissance, ou de la politique? Théologiques, 2(1), 81-101. https://doi.org/10.7202/602399ar
Résumé de l'article

À la fin des années 1970, le mouvement connu sous le nom de Créationnisme Scientifique s'était acquis suffisamment d'appui populaire pour entreprendre de faire adopter, dans chacun des états des États-Unis, un projet de loi légalisant l'enseignement d'une biologie alternative aux élèves des écoles publiques. Ce Créationnisme Scientifique alternatif s'opposait à l'option prédominante actuelle en faveur de l'évolution telle qu'enseignée par les biologistes scientifiques; dès lors surnommé l'Évolutionnisme Scientifique. Ces projets ont avorté au début des années 1980 et la tactique de ce mouvement s'est faite moins manifeste, canalisant ses énergies à la préparation d'enseignants pour travailler dans les écoles publiques et y enseigner une biologie créationniste. Néanmoins, le mouvement a eu des retombées importantes en dégageant un espace intellectuo-politique alternatif auquel d'autres fondamentalistes peuvent recourir, avec un sentiment de légitimité en matière de vérité et de connaissance.
Tous droits réservés @ Faculté de théologie de l'Université de Montréal, 1994

Ce document est protégé par la loi sur le droit d'auteur. L'utilisation des services d’Érudit (y compris la reproduction) est assujettie à sa politique d'utilisation que vous pouvez consulter en ligne.

https://apropos.erudit.org/fr/usagers/politique-dutilisation/ 
Théologiques 2/1 (1994) 81-101.

\title{
L'attaque religieuse contre la science : une bataille pour la définition de la vérité et de la connaissance, ou de la politique? *
}

\author{
Harvey B. SARLES \\ Cultural Studies and Comparative Literature \\ Université du Minnesota
}

\section{RÉSUMÉ}

A la fin des années 1970 , le mouvement connu sous le nom de Créationnisme Scientifique s'était acquis suffisamment d'appui populaire pour entreprendre de faire adopter, dans chacun des états des États-Unis, un projet de loi légalisant l'enseignement d'une biologie alternative aux élèves des écoles publiques. Ce Créationnisme Scientifique alternatif s'opposait à l'option prédominante actuelle en faveur de l'évolution telle qu'enseignée par les biologistes scientifiques; dès lors surnommé l'Évolutionnisme Scientifique. Ces projets ont avorté au début des années 1980 et la tactique de ce mouvement s'est faite moins manifeste, canalisant ses énergies à la préparation d'enseignants pour travailler dans les écoles publiques et y enseigner une biologie créationniste. Néanmoins, le mouvement a eu des retombées importantes en dégageant un espace intellectuo-politique alternatif auquel d'autres fondamentalistes peuvent recourir, avec un sentiment de légitimité en matière de vérité et de connaissance.

\section{$* * *$}

Par conséquent, dans le dernier chapitre de ce livre, le modèle de création courant est expliqué plus explicitement dans les catégories de la révélation biblique. Toute la question des origines et du

\footnotetext{
* Texte traduit par M. Jean-Claude Breton. Généreux remerciements à Janis Sarles
} pour sa critique du présent exposé. 
développement est replacée dans son contexte biblique et théologique correct, et l'élève est conduit vers une vision du monde complète, cohérente et satisfaisante, organisée autour du Créateur et Sauveur personnel, le Seigneur Jésus Christ.

Il faut souligner que cet ordre est adopté non pas parce que les informations scientifiques sont considérées plus fiables que la doctrine biblique. Au contraire, c'est précisément parce que la révélation biblique représente une compétence absolue et claire que la réalité scientifique, bien comprise, va fournir le même témoignage que celui des Écritures. Il n'y a pas la moindre possibilité pour les données de la science de contredire la Bible... $(15)^{1}$

\section{Préface}

Les créationnistes scientifiques s'efforcent d'impliquer les scientifiques dans un débat entre création et évolution aux États-Unis. Désireux d'insérer leurs idées dans le tissu de la pensée sociale, ils se concentrent particulièrement sur les écoles publiques et l'enseignement de la théorie de l'évolution. Comme les écoles publiques sont soumises aux lois publiques, le champ de cet affrontement comporte le changement des lois et la formation d'un groupe d'enseignants épousant cette position.

L'argumentation englobe la nature de notre être, de la connaissance, de la vérité et de la compétence, en même temps intellectuelle, théologique et politique. Ce mouvement est une entreprise pour ramener ces questions du domaine (plutôt) humain de la raison et de la recherche scientifique vers les textes bibliques interprétés dans ce cas par ceux qui s'appellent les Créationnistes Scientifiques.

La stratégie habituelle n'est pas d'argumenter contre la raison ou la science de façon ouverte et directe. Elle cherche plutôt à montrer que la création et la science sont toutes deux des formes de religion. Les créationnistes s'inquiètent de ce que les scientifiques, comme les gens d'avant le déluge au temps de Noé, attaquent le droit de Dieu à la vérité et à l'omniscience, et ils craignent sa colère. Il est donc temps de revenir à sa Parole.

1 Les numéros de pages non-identifiés renvoient à l'ouvrage de MORRIS, 1974. 


\section{Une brève présentation historique}

À la fin des années ' 70 , un pasteur protestant fondamentaliste de Minneapolis a adressé une lettre à quelques facultés de l'université du Minnesota. Les facultés visées étaient celles dont les programmes départementaux comptaient certains cours avec le mot évolution dans leur titre. Cela incluait l'anthropologie (où j'enseignais), la géologie, et plusieurs cours du département de biologie.

Cette lettre nous exhortait à ajouter un cours sur le Créationnisme Scientifique, pour accompagner et compléter nos offres de cours sur l'évolution, qui devraient être renommés Évolutionnisme Scientifique. L'évolution était manifestement l'objet d'une attaque venue d'une réécriture, d'une relecture et d'une remise en question. En d'autres mots, l'évolution, resituée dans un nouveau contexte, était comparable ou équivalente au créationnisme.

S'agissait-il d'une tactique pour introduire la religion dans les écoles publiques? Il apparaissait significatif que l'université du Minnesota soit de par sa charte une université publique; en fait, une école publique supérieure gérée par l'État du Minnesota et soumise à ses lois. Dans ces écoles, l'enseignement de la religion est de soi interdit par la loi, même si on peut y étudier le phénomène religieux dans des départements ou des cours de sciences religieuses ${ }^{2}$.

Mes collègues en anthropologie ont pour leur part manifesté peu d'intérêt, se contentant d'une acceptation tacite de ma préoccupation. La réaction commune était qu'il s'agissait de l'oeuvte d'un cinglé ou d'un fou. Dans l'esprit de la majorité, le procès Scopes de 1925 était incontestable: une victoire définitive de l'évolution, une fois pour toutes.

2 La majorité des collèges et universités des États-Unis entretiennent des liens avec une confession religieuse, même quand ils sont (apparemment) laïques. Il y a bien sûr plusieurs institutions d'enseignement supérieur qui sont directement rattachées à une confession religieuse et administrées par elle: les plus connues, peut-être, sont Georgetown et Notre-Dame (Catholiques) et Brigham Young (Mormons). Les sciences religieuses consistent habituellement en un programme où des cours en religions comparées sont offerts, sans qu'une direction ou une théologie ne soit proposée; il n'y a pas de chapelle ni de services religieux, et ainsi de suite. Jusqu'à l'époque de Darwin (vers 1860), presque toutes les universités américaines étaient rattachées à une Église, et il y avait bien peu de recherche scientifique. Les universités publiques et la science se sont épanouies seulement après certe époque (METZGER, 1955). 
Nous étions cependant dans les années '70. C'était l'époque des sectes: des moonistes, des disciples de Hare Krishna, une décennie après l'engouement américain pour les drogues, les replis dans les communes, l'épisode destructeur de la famille Manson, Jonestown; l'époque des attirailes étranges et des festivals sur les campus universiraires, où toutes sortes de groupes chamans et mystiques sont apparus. Le livre Snapping (Conway et Siegelman, 1978) étudie cette période. Il essaie de montrer comment ceux qui sont en recherche peuvent être endoctrinés soit par la Scientologie, EST, ou AMWAY, ou n'importe quel groupe qui semble promettre quelque chose de religieux. Des récits de déprogrammation d'anciens membres de sectes commençaient à paraître. Que cherchent-elles vraiment ces personnes qui adhèrent à des sectes, retournent au fondamentalisme et aux littéralismes ${ }^{3}$ ?

Je me suis entretenu avec des collègues, des voisins et des étudiants qui ont été éduqués dans la ligne du fondamentalisme protestant, mais qui sont passés à des positions plus libérales (leur formule). Leur discours portair sur le livre de l'Apocalypse, et sur leurs efforts pour se détacher de leur éducation religieuse étroite.

Cette époque et ces échanges entamaient ma vieille conviction que nous devions (tous) devenir de plus en plus rationnels. Les États-Unis des années '70 me rappelaient une longue période de travail sur le terrain dans le Mexique autochtone, où j'avais pour la première fois rencontré mystiques et mysticisme, chamans et voyants, des guérisseurs en compétition avec des médecins scientifiques occidentaux. Intéressé par la lettre du pasteur, je me suis mis à l'étude du livre que sa lettre recommandait sur le Créationnisme Scientifique par Henry M. Morris (1974), directeur de l'Institut pour la recherche sur la Création de San Diego, Californie.

C'est alors qu'eut lieu dans la plupart des États la tentative d'imposer légalement l'enseignement du Créationnisme Scientifique dans les écoles publiques, tentative qui échoua finalement en Louisiane au début des années ' 804 .

3 Pour une synthèse explicative approfondie de ce mouvement à cette époque, voir MARTY, 1981.

$4 \quad$ Il y a eu de fait un tel projet de loi en vue d'enseigner le Créationnisme déposé à la législature de l'État du Minnesota, en 1979. Nombre d'entre nous, les professeurs avec quelques enseignants en biologie au secondaire (des comités de correspondants se sont formés partout dans le pays), le Père Hunt du Centre Newman de l'Université du 
À l'heure actuelle, le mouvement du Créationnisme Scientifique échappe au regard public des cours ou des législatures d'État. Le mouvement est toutefois actif à former des enseignants en biologie dans les écoles secondaires de tout le pays. Il a obtenu que plusieurs collèges soient reconnus pour former ces enseignants en biologie selon la visée créationniste. Sa perspective est devenue une composante d'un mouvement fondamentaliste plus large, regroupé dans la Coalition Chrétienne.

\section{Le contexte}

Les questions sous-jacentes au débat création-évolution couvrent un très large éventail. Elles comprennent 1) les questions théo-politiques de la séparation de l'Église et de l'État; 2) les ressemblances et les différences entre la culture comprise comme un parti-pris pour le passé ou pour une vision généreuse du présent; 3 ) les questions métaphysiques sur la vérité et la compétence; 4) des questions existentielles et psychologiques sur la destinée personnelle qui ont des retombées décisives sur les concepts d'être et d'histoire; et, 5) des particularités sur ce que l'idée d'équité signifie aux États-Unis, ce qui a permis au créationnisme de défier la science.

Ce débat intervient surtout dans le cadre légal et historique des ÉtatsUnis. Aussi, une certaine compréhension de ces questions dans leur histoire culturelle et religieuse est utile pour voir comment les raisonnements résonnent aujourd'hui, aussi bien que pour connaître les situations d'où ils tirent leur sens et leur force.

\subsection{Les questions théo-politiques}

La première situation porte sur la codification légale du conseil de Matthieu 22,21 où il est dit qu'il y a séparation entre l'Église et l'État. Le premier amendement de la constitution des États-Unis renferme deux paragraphes à propos de la religion: a) l'assurance de la liberté du culte religieux pour tout le monde; b) l'interdiction d'établir une religion d'État.

Les créationnistes prétendent que les évolutionnistes sont, en réalité, en train de présenter des idées religieuses au nom de la vérité

Minnesota, et quelques leaders religieux de tendance libérale, ont témoigné contre le projet de loi, qui a été battu de peu au comité sur l'éducation de la chambre des représentants du Minnesota. Le Père Hunt établit la distinction entre foi religieuse et théorie scientifique dans sa déposition contre l'adoption du projet de loi. 
scientifique, et qu'ils ont ainsi fondé une religion d'État. Ils soutiennent que les élèves des écoles publiques ne sont pas réellement libres de mettre en doute l'évolution dans les cours de biologie. Il s'en suit que la liberté de religion est supprimée. Pour les créationnistes, il n'y a ni science ni raison. Il n'y a que la religion. Pour ceux qui séparent les royaumes parallèles de la foi et de la raison, cette volonté de faire de la science une religion fait partie d'une tentative de miner la démocratie et de transformer le pays en une théocratie.

\subsection{Les guerres de culture}

Le fait d'émousser et de camoufler la séparation de l'Église et de l'État relèvent de certaines particularités de l'histoire américaine, et surtout de leur impact sur la situation actuelle. Cela reflète en partie les questions de l'identité nationale qui s'est, pour une large part, précisée dans l'opposition américaine à l'empire du mal du communisme, mais qui n'est plus perçu comme une menace. Nous nous retrouvons renvoyés à une sérieuse quête de notre identité, après une époque où nous nous sommes définis principalement en fonction de ce à qui et à quoi nous nous opposions. En ce sens, la démocratie et le capitalisme se sont confondus. Le capitalisme semble la voie de l'avenir, avec des principes démocratiques en arrière-plan. Une nouvelle querelle culturelle semble s'annoncer, détournant l'attention des principes du gouvernement pour la braquer sur la compétition dans un monde industriel.

Comment cela est-il arrivé? Aux États-Unis, le thème de la théologie et de la démocratie a une histoire longue et compliquée. La plus grande partie de l'histoire concerne le désaccord protestant à l'égard de religions organisées et/ou d'État. Ce sentiment est bien illustré dans le Pilgrim's Progress de Bunyon (1987), où chaque individu emprunte son propre chemin vers la vérité. Sa théologie (d'origine européenne) s'est compliquée en raison de la géographie et des cultures qui sont arrivées ici en vagues successives: les protestants, qui sont aussi ethniquement des Anglais, des Écossais, des Allemands et des scandinaves, sans oublier les afroaméricains du post-esclavagisme; des catholiques romains, qui sont en même temps des Irlandais, des Allemands, des slaves, des hispaniques et des méditerranéens; des Juifs, en même temps des Allemands, des Russes, des Polonais.

L'histoire religieuse américaine se mêle à des considérations socioéconomiques et ethniques, accentuées par la raison du premier arrivé et la loi du plus fort. Chaque groupe qui a atteint un certain degré d'identité nationale et de succès s'est donc opposé, à son tour, pour des raisons théo- 
culturelles, et dans des débats sur ce qu'est une vraie personne et sur ce que cela signifie (Lipset, 1969), aux arrivants suivants: un anti-catholicisme, un anti-sémitisme et à l'heure actuelle un anti-féminisme et un antipersonnes de couleur.

À l'intérieur au moins des populations d'extraction européenne ces conflits ont toutefois vraiment diminué au cours de la dernière génération (Christopher, 1989). À leur place, une sorte de consensus à double polarité est en train de se développer au sujet des questions religieuses, ce que Hunter définit comme des guerres de culture (1991).

Sur cette vaste toile de fond, la bataille créationniste-évolutionniste s'inscrit surtout dans une perspective blanche et protestante. Mais cette perspective s'est récemment élargie pour devenir une sorte de conservatisme économique théo-politique, pour ceux qui adoptent une foi très fondamentaliste à propos d'un ensemble de questions socioéconomiques. Ils se disent pour Dieu, l'Église et la famille, et contre le crime et les progressistes. La controverse autour de l'avortement, en particulier, contribue à rallier ceux qui ont adopté une position ferme en faveur d'une perspective (religieuse) conservatrice, où le partage du fait de croire supplante les différences confessionnelles. Une bonne part de cette soi-disant bataille culturelle porte sur des questions de valeurs et d'absolus. L'opposition au relativisme religieux et/ou moral devient un emblème de patriotisme. Dans ce contexte, le Créationnisme Scientifique sert surtout à dégager un large espace à un ralliement des forces conservatrices. Reste à voir si cette théo-politique va tenir au-delà du débat sur l'avortement.

\subsection{La vérité et la compétence}

Dans ce contexte, le créationnisme présente une théorie antiprogressiste tout en abordant les enjeux métaphysiques de vérité et de compétence. Cette théorie particulière voudrait nous ramener à une approche littérale des textes bibliques, tout en prétendant en même temps offrir une alternative précise aux manières libérales, scientifiques et modernes d'établir la vérité. Sous la bannière de l'anti-progressisme, ce fondamentalisme littéral semble être tout au plus une autre forme de conservatisme, ou une autre justification de celui-ci.

Elle propose un ennemi identifié: l'humaniste séculier, qui voudrait sortir la prière des salles de cours des écoles et qui croit dans la vérité de 
la science, comme antithèse à la vérité de Dieu ${ }^{5}$. Du point de vue du conservatisme et du progressisme, l'humaniste séculier se caractérise moins par son intérêt pour la science et la raison que, surtout, pour son opposition à Dieu. La tentative humaniste historique pour s'approcher de l'objectivité et de la raison est réévaluée et identifiée comme une religion anti-religieuse. Le concept de progrès scientifique est situé à l'intérieur du progressisme en autant que ce dernier s'oppose au maintien de la perspective religieuse.

Le Créationnisme Scientifique se préoccupe de la définition et du contrôle de la vérité et de la compétence. Aussi, sa contribution à la discussion publique s'est surtout attachée au système scolaire public, qui semble enseigner et adopter une position scientifico-progressiste particulière au sujet de la connaissance et de la vérité. Les créationnistes se sont beaucoup intéressés au débat autour de l'école publique aux ÉtatsUnis.

Les écoles publiques sont vues comme des faillites, et les accusations fusent de partout. Les mots mêmes du début du livre de Morris ouvrent le débat: "La tendance largement répandue au cours des dernières années d'ouvrir de nouvelles écoles chrétiennes privées a été stimulée pour une large part par la faillite des écoles publiques à entretenir une objectivité académique et philosophique.» Et il continue: «Au nom de la science moderne et de la séparation de l'Église et de l'État, la Bible et la religion théiste ont été de fait éliminées des programmes, et une religion non-théiste de l'humanisme séculier évolutionniste est devenue... la religion d'État officielle encouragée dans les écoles publiques» (iii).

5 L'humanisme séculier est un nom que le Créationnisme Scientifique utilise pour envelopper tous ses adversaires. Il est bien difficile de savoir s'il y a effectivement des humanistes séculiers aux USA. Le mouvement de réaction s'est concentré autour des publications du Professeur Paul Kurtz, philosophe émérite au SUNY (State University of New York), à Buffalo. Il édite deux revues, Critical Inquiry et le Skeptical Inquirer, qui abordent toures les positions anti-scientifiques, du fondamentalisme religieux au ESP et autres chimères scientifiques. Il est aussi l'éditeur de Prometheus Press, dont la liste de publications témoigne de ses positions. Parmi d'autres qui ont revêtu les couleurs de l'humanisme (séculier), on compte l'American Humanist Society (société humaniste américaine), la Ethical Culture Society (société de la culture éthique) et sa filiale la Humanist Institute, (institut humaniste), dont je suis membre du corps professoral. Ce que l'humanisme a d'élan aujourd'hui (ce n'est pas beaucoup!) prend son point de départ de la position anti-humaniste du créationnisme. 
Le cadre intellectuel du Créationnisme Scientifique semble bien éloigné de la Realpolitik. Il se présente comme une querelle académique plutôt que comme un débat politique. Mais, comme c'est souvent le cas dans le monde (post)moderne, les principaux objets de débat finissent par être de l'ordre de la lecture, de l'interprétation et de la compétence. Sur ce point, le Créationnisme Scientifique propose une façon alternative de connaître. Ses principaux textes sont des extraits des évangiles du Nouveau Testament et des apocryphes. Ils privilégient particulièrement l'Apocalypse. Ces textes sont donc reçus au niveau de leur sens obvie donc, en vue d'une re-lecture du livre de la Genèse, le texte-clef pour la création et l'histoire de Noé.

Il est également d'une grande importance dans cette discussion au sujet de la création d'aborder le thème de l'an mille annoncé avec tant de puissance dans l'Apocalypse: l'apocalypse, l'holocauste, le retour du Messie, notre évasion de la terre, et le retour de nos âmes au ciel.

En l'an 2000 ou autour, (ce qui est maintenant à portée de la main), nous pouvons prévoir que bon nombre de fondamentalistes vont s'énerver à propos du soi-disant ravissement au cours duquel la divinité reviendra sur terre. Les âmes de ces chrétiens qui sont nés de nouveau vont être ramenées au ciel, tandis que les autres seront condamnées à un authentique enfer sur terre. Semblable agitation a eu lieu à la veille de l'an 1000, alors que de nombreux chrétiens sont allés à Jérusalem pour attendre la seconde venue, mettant sur pied les Croisades et modifiant radicalement le monde occidental. Elle a resurgi encore autour de l'an 1500, que les fameuses sculptures de l'Apocalypse de Durer ont voulu célébrer. Nous pouvons nous attendre encore à la même chose, bientôt!

Par le recours à ces textes comme à des idées et/ou des positions de base, le Créationnisme Scientifique est une re-lecture néotestamentaire singulière de la Genèse, avec un accent spécial mis sur le déluge ( $\mathrm{Gn}$ 6-9). Comme ce texte, le créationniste scientifique met l'accent sur une hydrologie du déluge. Cela implique la compréhension de la vie comme un moment du retour de l'âme au ciel, et un effort pour expliquer pourquoi nous sommes ici sur terre, ce que nos vies signifient, et ainsi de suite. Mais une place spéciale est réservée à la préoccupation de l'hybris humain de chercher toute connaissance grâce à la science. Comme une 
large part de son histoire lui est commune au catholicisme, une large part aussi de ce récit est familier; mais beaucoup aussi est différent ${ }^{6}$.

Quels textes sont mis en évidence? Comment sont-ils interprétés et compris? Cela est décisif. Nous savons que cette question de la lecture, de la littéralité et de l'interprétation est très ancienne dans le monde chrétien. De fait, la grosse part du travail de base en vue de la fondation du Catholicisme par Augustin (1958) aborde ces questions d'exégèse et d'interprétation dans le détail, et plaide en faveur d'une classe de prêtres instruits qui seront capables de lire le texte biblique avec une compétence bien intentionnée et prudente dans le cadre de la communauté (charité), par opposition à une lecture individuelle et littérale.

Le Créationnisme Scientifique, comme d'autres fondamentalismes, représente un essai de retour à une lecture littérale des textes bibliques, et les différentes formes de fondamentalisme croient à la soi-disant inerrance de la Bible ${ }^{7}$. Sous plusieurs aspects, notre temps est une reprise du temps d'Augustin, un temps où décider quels textes lire et comment les interpréter, qui a la compétence pour trancher ces questions, et sur quels appuis. Pour Augustin, il s'agissait d'un clergé intellectuel qui étudierait et qui interpréterait, au sein - comme cela est arrivé - d'une institution: l'Église. Pour le littéraliste, le but - au moins en ce moment - consiste à revenir à la compétence des textes bibliques et des lecteurs individuels. Et

6 L'utilisation du mot récit/story peut soulever des objections de la part des Créationnistes Scientifiques. Cela devrait montrer les barrières qui séparent ceux qui comprennent la Bible comme inerrante et à prendre à la lettre, et les autres qui acceptent la nécessité d'une interprétation.

7 Ceux d'entre nous qui suivent les raisonnements d'Augustin (1958) et/ou qui abordent d'une manière critique les problématiques de la compétence et de l'interprétation rejettent la possibilité de l'inerrance pour plusieurs raisons, la moindre n'étant pas qu'il est difficile de comprendre la pensée d'auteurs - qui ont vécu à d'autres temps et dans d'autres cultures que les nôtres - dans leurs propres mots. Bien plus, comme le dit Augustin, il y une sémantique - des signes naturels et conventionnels - qui recourt à une théorie (34-7), au contraste (37), aux contradictions et aux ambiguïtés (38), à des langues différentes (46), des traductions (48-9), etc. De fait, l'histoire de l'étude de l'origine des langues a été stimulée pour une bonne part par le désir de redécouvrir la langue adamique, le soi-disant vrai langage de Dieu; une référence à Gn 2,19 où Adam est instruit du vrai (=divin) nom des animaux; un autre des textes majeurs du Créationnisme Scientifique. «Le Seigneur Dieu modela du sol toute bête des champs et tout oiseau du ciel qu'il amena à l'homme pour voir comment il les désignerait. Tout ce que désigna l'homme avait pour nom 'être vivant'» (voir HEARNE, 1986). 
la pratique même de l'exégèse est enterrée. Même si le combat pour la compétence est bien ancien, il réapparaît encore aujourd'hui dans ces contextes théo-politiques rendus plus compliqués par l'histoire, la culture et les pratiques de conscience de soi du révisionnisme.

Les principales questions intellectuelles que le Créationnisme Scientifique aime mettre de l'avant portent sur la nature humaine: en particulier comment nous sommes, pourquoi nous sommes ici sur terre et comment retourner au ciel, auquel nous (nos âmes) appartenons. Certaines questions sont communes à tout le christianisme ou à sa grande majorité, en particulier le souci (néo-platonicien) autour des affirmations augustiniennes à propos de la chute humaine sur terre. L'idée persistante est que nous, comme âmes, appartenons au ciel et sommes sur la terre d'abord en raison d'une punition, notre but étant de ramener nos âmes au $\mathrm{ciel}^{8}$. La compréhension même de la mort est celle d'une punition pour notre orgueil, notre hybris et notre désobéissance. L'orgueil et l'hybris sont particulièrement funestes aux yeux des Créationnistes Scientifiques!

Même si quelques problèmes sont partagés plus étroitement avec d'autres fondamentalistes protestants - en particulier la lutte contre l'évolution et contre l'idée de changement ${ }^{9}$ - le Créationnisme Scientifique met au coeur de ses théories la question de l'hybris humanoscientifique. Il croit, surtout, à la théorie d'une terre jeune (plus de 6000 ans, environ), à un monde organisé autour de la terre (géocentrique) fait

8 Cette question refait surface, en particulier à notre époque, à l'occasion du débat autour de l'avortement. Les tendances universalisantes de la pensée grecque sont particulièrement importantes pour comprendre la pensée chrétienne, surtout dans son contraste avec la pensée juive antérieure qui est, à mon avis, clairement caractérisée comme plus existentielle; e.g. le récit d'Adam et Ėve est compris par la majorité des Juifs comme une description de l'expérience de tout couple amoureux à la découverte de la nudité l'un de l'autre, tandis que la ré-interprétation chrétienne soutient que le récit d'Adam et Ėve porte sur la condamnation de tous les humains parce que Ève (une femme) a désobéi à Dieu au jardin d'Éden (FELDMAN, 1968). Au moment de la rédaction du présent exposé, les fondamentalistes et l'Église se sont ralliés à propos de la question de l'avortement, mais sur aucune autre question; une association inconfortable?

9 Les fondamentalistes protestants ne font pas de distinction entre les domaines de la foi et de la raison. La vie est fondée sur la foi, et la raison est utile en autant qu'elle sert la foi. À mon avis, le créarionnisme et d'autres formes de lectures littérales des textes bibliques nous conduisent directement à une théocratie, supprimant les idées qui séparent l'Église de l'État, la foi de la raison. Dans cette perspective, le fondamentalisme n'a tout simplement pas de place pour l'humanisme chrétien. 
pour nous les humains; où toutes les espèces ont été créées dès le début; où il n'y a pas eu ni ne peut pas y avoir de changement. Les six jours de la création sont exactement cela: six jours, écoulés une fois pour toutes.

Le principal penseur de ce mouvement, Henry Morris, est un ingénieur en hydrologie détenteur d'un diplôme (ironie du sort?) de l'université du Minnesota ( $\mathrm{PhD}$., à la fin des années '30), et cela n'est pas un hasard. Parce que le récit du déluge, l'embarquement de chaque espèce dans l'arche par Noé, atteste pour les créationnistes que toutes les espèces ont été créées par Dieu... au commencement; aussi il n'y a pas eu (ne pouvait y avoir) d'évolution. Toutes les espèces qui existent sur terre ont été créées à ce moment-là, et sauvées par l'arche de Noé. Cette idée anti-évolutionniste sera utile pour soutenir l'idée du retour du messie lors du prochain millénaire, faisant clairement échec à une compréhension linéaire de l'histoire ${ }^{10}$.

Une partie de cette querelle a déjà eu lieu au XVIII ${ }^{\mathrm{e}}$ siècle, avant que les principes modernes de géologie ne soient bien compris. Il y eut un grand débat, en partie encore pertinent, à savoir si l'histoire de la terre à partir des premiers temps jusqu'à son état actuel était plus ou moins linéaire et uniforme (uniformitarianisme), ou si la terre s'était surtout constituée à partir d'événements subits (catastrophisme): déluges (!), météores, volcans. Dans ce cas-ci, la pensée tend à être du genre ou bien/ou bien, comme le plus souvent lors de ce débat, même si les créationnistes manifestent bien peu d'intérêt pour l'histoire des idées. Le déluge de Noé tient lieu de preuve pour le Créationnisme Scientifique que le monde est conduit surtout par des catastrophes, s'occupant aussi de nos efforts faustiens/diaboliques pour défier la volonté de Dieu et sa connaissance omnisciente. À chaque fois que nous (i.e. la science, cette foisci) contreviendrons, la colère de Dieu nous tombera encore dessus.

Le premier objet de recherche qui pourrait rétablir les faits dans cette querelle est le livre des fossiles. Le point de départ de la charge du créationnisme contre l'évolutionnisme porte surtout sur la géologie: les fossiles, les méthodes de datation et ainsi de suite. L'entreprise vise à prouver que le livre des fossiles atteste un catastrophisme évident, qu'il n'y a pas eu d'évolution d'une espèce à une autre, aucun état intermédiaire,

10 L'idée du retour messianique implique que tout interstice ( 1 ou 2000 ans) sera annulé, comme si aucun temps ne s'était écoulé entre sa première venue et son prochain retour sur terre. Toute l'histoire institutionnelle et culturelle serait donc comme si elle n'avait jamais existé. 
aucun chaînon manquant. Les collisions de météores sont préférées à la stratigraphie et au carbonne-14 comme méthodes de datation.

Le deuxième champ de bataille est celui de la biologie: savoir si nous avons évolué ou sommes venus des bêtes, de créatures inférieures, ou si nous avons été faits immédiatement à l'image de Dieu comme dans Gn 1,26. Si, s'interroge Morris, nous sommes comme des bêtes, alors nous devrions agir comme des bêtes; pas vraiment capables de connaître Dieu, et à jamais incapables de retourner au ciel.

Pour les créationnistes, notre existence même comme êtres humains est en jeu dans ce débat. Les questions autour de la sexualité, du péché et de l'âme sont prépondérantes. Une partie de la querelle s'intéresse à l'anatomie comparée, au fait de partager une morphologie et un comportement avec d'autres espèces, en vue de démontrer que les humains sont spécifiquement uniques. Bien sûr, le débat tient en partie au fait que les travaux récents en ethnologie, dans l'enseignement aux singes d'un langage par signes, et ainsi de suite, ont remis en question la singularité humaine, y compris le langage, la rationalité et la connaissance (Sarles, 1985).

Le dernier champ contextuel du débat intellectuel, un champ qui commence à peine à apparaître (1993), est celui de l'anthropologie. Son questionnement porte sur ce qu'est la nature humaine, le langage, la culture, la religion (179). Le thème des origines humaines est particulièrement délicat: le langage, le sens, et notre connaissance de Dieu. Mais toute la méthode comparative, confrontant les humains avec d'autres espèces pour découvrir des ressemblances et des différences, l'idée d'un rapport aux autres espèces, est anathème ${ }^{11}$. Dieu a fait le monde pour nous, nous seuls, à son image. L'humanité est première, les bêtes sont là pour que nous en disposions; certainement pas pour que nous leur soyons comparés.

$11 \grave{A}$ mon avis, la question fait surface seulement maintenant dans le cadre de ce que j'appelle «Des aspects de la crise dans le sens», une montée du nihilisme, une perte d'identité, un retour souhaité à l'ethnocentrisme et au nationalisme surtout en lien avec la chute du bloc soviétique (SARLES, $1993 \mathrm{msl}$ ). Plus concrètement, Motris s'inquiète de ce que si quelqu'un croit à l'évolution à partir des bêtes, il se «comportera comme une bête, soit en luttant agressivement lui-même pour sa suprématie, soit en se soumettant aveuglément à des leaders agressifs» (iii). 


\subsection{Les enjeux existentiels}

L'élan existentiel-psychologique du créationnisme, et pour une large part du fondamentalisme, semble être soutenu par la peur de la mort (un enjeu de taille), mais surtout par un doute persistant à propos de l'existence même de l'individu. La préoccupation du créationnisme, comme il a été dit, porte sur les textes de la création du monde et des espèces, dans la Genese. Dans le cadre de leur argumentation ou bien / ou bien, le monde a été créé dans les six jours de la création comme exposé dans $\mathrm{G} n$ 1. La création a eu lieu durant ce temps. Le monde est essentiellement statique. L'éternité de Dieu, de nos âmes, est la réalité. Si notre être même est une erreur née de la désobéissance et du péché, alors la vie ellemême demeure un casse-tête risquée ${ }^{12}$.

De la même manière, la réalité et la compréhension de l'histoire deviennent douteuses. En proposant ce cadre de référence, il est important de souligner que l'approche catastrophique de l'être implique une approche alternative, ou bien ou bien, de l'idée même d'histoire et de la construction du temps. Je fais ici une distinction entre un sens linéaire, previsible, de l'histoire, et un sens prophétique. Le créationnisme est à l'évidence une tentative pour nous ramener à un sens prophétique de l'histoire. Le retour millénariste du messie va littéralement effacer (la mémoire) les 2000 ans d'intervalle. Les concepts d'histoire et de culture n'ont pas de place dans cette pensée. En un sens, la charge créationniste contre la raison contribue à étendre son pouvoir.

\subsection{L'équité}

En dernier lieu, la question de l'appellation scientifique comme qualificatif du créationnisme ou de l'évolutionnisme se laisse comprendre au mieux, de l'avis de Nelkin (1977), en référence au concept américain d'équité, tel qu'utilisé dans les média de communication. Selon ce concept, tout point de vue doit être comparé à son contraire à la radio ou à la télé et recevoir un temps égal, au nom de l'équité. De même que toute position, disons des Démocrates, mérite de recevoir la répartie des Républicains, toute position peut être comprise comme ayant son antithèse: une sorte

12 Je ne crois pas exagérer en suggérant que la décision de proposer le créationnisme - et plusieurs autres retours fondamentalistes aux textes anciens - est motivée pour une large part par l'inquiétude à propos de la précarité de l'existence (SARLES, $1992 \mathrm{~ms} 2)$. 
d'antithèse équivalente ${ }^{13}$. Il y a toujours des positions adverses, et chacune devrait être entendue, en particulier, pour les créationnistes, par les élèves des cours de biologie. C'est pourquoi la religion et la science se voient reconnaître un statut équivalent et contradictoire. De la sorte, les démarcations et les champs respectifs de la foi et de la raison s'estompent, de même qu'entre l'Église et l'État, et ailleurs.

Le soin mis pour opposer le créationnisme (une notion clairement religieuse) à l'évolution (une idée raisonnée, scientifiquement établie) est très habile et éventuellement puissant. Il oppose évidemment la religion à la science, mais il le fait au nom d'une idée communément acceptée, une coaptation, en langage moderne. Les deux options deviennent scientifiques, alors que les deux sont des questions de foi. À mesure que la religion devient une sorte de science, la science devient une question de foi. De fait, les créationnistes soutiennent que la création et la science sont toutes deux objets de foi; les deux sont des croyances, les deux sont des religions. Rien n'existe que la religion, théiste ou non-théiste. La science, comme recherche de la vérité et de la nature des choses, dégringole d'une pente glissante. La raison et la logique n'existent qu'au service de la foi.

Ils affirment de plus qu'ils ne peuvent pas se tromper (15), alors que la science reconnaît régulièrement (comme une force) qu'elle formule des hypothèses et propose des démonstrations provisoires, une faiblesse du point de vue des créationnistes. Cela contribue, à mon avis, au travail de sape actuel du nihilisme à l'égard du sens, de la science, de la possibilité de quelque sorte de vérité objective, et conduit vers une destruction et une crise réelle du sens (Sarles, $1993 \mathrm{~ms} 1$ ).

\section{L'hybris de la science}

Une bonne part de la réaction du Créationnisme Scientifique consiste à s'en prendre au dogmatisme et aux prétentions d'omniscience quasi divine que certains scientifiques déterministes mettent de l'avant au nom même de l'idée de science, une tentative progressiste pour contrôler la nature, et dès lors la nature humaine. Morris réagit amèrement à des prises de position comme celles-ci (199):

13 Il est aussi important de remarquer que Morris, et d'autres en nombre croissant, ont adopté des pratiques académiques dans leur écriture, leurs références, leurs citations, etc., donnant à leurs travaux l'apparence des textes universitaires, alors qu'ils sont des pamphlets religieux. 
En raison de la capacité sans précédents, à la fois offerte et contrôlée par nous, de prévoir à long terme, nous pouvons, en assurant et en améliorant notre position, éviter de plus en plus les faux-pas de la nature aveugle, nous dérober à ses cruautés, perfectionner nos propres natures et améliorer nos propres valeurs (MULLER, 1958).

La seule caractéristique de l'homme parmi les animaux est sa capacité de diriger et de contrôler sa propre évolution, et la science est l'outil le plus puissant à cet effet. Nous sommes le produit de deux sortes d'évolution, biologique et culturelle. Nous sommes ici en raison du même processus de sélection naturelle qui a produit les autres plantes et animaux; il s'est amorcé il y a environ un million d'années avec nos ancêtres hominoïdes fabricants d'outils (HOAGLAND, 1964).

Ce à quoi Morris répond: "Cette croyance que l'homme est capable de contrôler l'évolution à venir est tout simplement un autre indice du fait que l'évolution elle-même est une religion" (200). Même si nous pouvions contrôler l'évolution à venir, sa direction dépendrait fortement de jugements de valeurs, donc de la religion. Pour Morris, ces affirmations sont un retour à $G$ n 6,5 où l'homme devient à nouveau méchant, son coeur ne pensant qu'au mal, et la promesse de Dieu de le détruire encore une fois imminente. La dernière fois, seul Noé (et sa famille) a survécu. Qui survivra cette fois-ci? - la question suprême de l'existence certainement pas les évolutionnistes, les scientifiques ou leurs disciples.

Morris interroge: «... comment un processus contingent et impersonnel peut-il produire un animal complexe, non-contingent, et doté d'une conscience personnelle et de principes moraux à partir desquels il peut formuler tels et tels plans et jugements? " (200) Il y a une lacune dans ce récit évolutionniste, et Morris entend bien y remédier.

La théorie de la création des Créationnistes Scientifiques est présentée dans le dernier (VIII) chapitre de Morris: La création selon l'Écriture. Il y a trois parties au récit de la création. En résumé, 1) «les six jours de l'unique création; 2) la rébellion de l'homme et, en conséquence, la malédiction de Dieu sur tout le monde de l'homme; 3) le déluge destructeur du monde à l'époque de Noé, qui a largement laissé le monde nouveau sous le régime de l'uniformité naturelle» (215). Tout doit être étudié et compris à l'intérieur de ce cadre. Dieu a fait les espèces des créatures comme Il les voulait... et elles doivent demeurer telles.

La stratigraphie géologique doit se comprendre en référence au déluge. Le réseau des fleuves de l'Éden n'existe plus présentement sur terre, emporté par le déluge, et encore, et encore. Tout cela conduit à la 
conclusion: «... si la Bible et le christianisme sont un tant soit peu vrais, les âges géologiques doivent être tout à fait rejetés" (255).

Comme dans beaucoup d'autres présentations d'un système religieux, l'idée que se fait Morris du Créationnisme Scientifique prend très au sérieux ses postulats, textes et croyances et elle les exploite complètement. On doit lire cet écrit et ses autres textes pour atteindre à une perception plus complète de sa théologie. Elle est bien pensée et exposée, même si elle résonne étrangement pour ceux d'entre nous qui vivons dans les perspectives héliocentriques modernes, de l'interprétation, et d'une vision du monde plutôt rationnel-scientifique, où les mondes de la foi et de la raison sont distingués.

\section{Conclusion}

Depuis le début des années ' 80 , au moment où les projets de loi en faveur de l'enseignement du Créationnisme Scientifique dans les cours de biologie du secondaire (et dans les collèges publics) ont été défaits dans les législatures ou les tribunaux des différents États, la présence active du Créationnisme Scientifique s'est faite moins voyante et dramatique. Mais le mouvement continue, et il se donne lentement une sorte de pouvoir, un pouvoir de définition er de présence. Quel pouvoir...?

Dans des débats dans la majorité des universités et collèges importants, Duane Gish (un proche collaborateur de Henry Morris) accepte d'affronter dans une discussion n'importe quel évolutionniste (scientifique) consentant. Et, dans le style de débat où Gish s'impose par sa rhétorique élégante, il s'en tire très bien. Des milliers d'étudiants et de professeurs de l'enseignement supérieur ont été témoins de semblables débats, et ils en sont ressortis plus tout à fait convaincus que la science et l'évolution soient tout à fait vraies. Là où il y a eu un débat organisé, le créationnisme (Gish) a tenu son bout. Il a tout au moins convaincu la majorité de son auditoire d'être sympathique à son droit d'avoir ses opinions, au moins, pour des raisons d'équité. Que l'auditoire ait désserré ou non son attachement à la science et aux idées scientifiques, il a commencé à croire qu'il y a quelque chose d'acceptable dans les deux projets.

La vérité scientifique ne peut pas simplement éconduire Gish ou le Créationnisme Scientifique, du moins dans le cadre d'un débat. Et comme la majorité d'entre nous avons accepté nos perspectives scientifiques de façon moins que critique, et que nous sommes devenus des mordus de la télé, nous avons tendance à être bienveillants, surtout quand nous sommes en face de scientifiques dont le dogmatisme est avivé par le débat. 
Peut-être ne sommes-nous pas critiquement conscients de comment nous recevons les théories et les résultats de la pensée scientifique. Par exemple, une large part de la programmation que nous visionnons souvent à la télé publique, les conceptions et les descriptions de différentes espèces animales, sont présentées en référence implicite aux vues évolutionnistes. Les échanges publics en matière d'astronomie (en particulier la NASA), de géologie, de satellites, de taches solaires, de réchauffement global, de progrès technologique et ainsi de suite, acceptent purement et simplement la science et la pensée évolutive comme claires et évidentes.

Selon toute apparence, l'immense succès de la science et sa simple évidence semblent nous avoir autorisés à appuyer son emprise sur notre pensée. Il est loin d'être évident que tout ce savoir intervient dans la discussion du créationnisme-évolutionnisme sur la place publique ${ }^{14}$. Dans les écoles secondaires elles-mêmes, il ne reste que (selon les apparences) bien peu d'enseignement de la théorie évolutive dans les cours de biologie. En ce sens, le créationnisme, et plus généralement le fondamentalisme, a été très efficace. Cela tient pour beaucoup, et directement, au fait que la concurrence pour les revenus des manuels scolaires a tendance à amener les éditeurs à banaliser la majorité des manuels du secondaire, et à éviter les sujets de dispute.

Nous sommes devenus très mêlés au sujet des frontières entre la vérité, la compétence, la célébrité et le succès dans notre univers de télévision. Au Minnesota, par exemple, il y a des tentatives en vue de censurer presque 100 livres par année, et de les éliminer des bibliothèques des écoles publiques. Plusieurs de ces essais de censure réussissent. C'est dire qu'il y a plusieurs groupes (parentaux ou religieux) qui examinent les livres. Plusieurs parmi eux sont des fondamentalistes d'allégeances différentes. La majorité s'oppose à l'évolution pour n'importe quel prétexte parce que, à leurs yeux, elle est opposée à Dieu.

Comme nous l'avons déjà affirmé, le mouvement pour introduire le créationnisme dans les cours de biologie s'est déplacé, depuis le début des années '80, de la scène publique et légale vers la formation de professeurs du secondaire, qui enseignent de fait le créationnisme comme étant de la biologie. Par exemple, le Nothwest College (autrefois le

14 Jusqu'où le doute à propos du système occidental influence aussi dans nos vies la suprématie de la raison et de la science, les questions de subjectivité et les charges contre le soi-disant projet des Lumières, demeure aussi obscur. Que ces deux mouvements se renforcent l'un l'autre est évident. 
Northwest Bible College) dans la banlieue de Minneapolis produit maintenant des enseignants diplômés, dont on peut présumer qu'ils obtiennent des postes dans diverses commissions scolaires. Il y a d'autres collèges semblables dans le pays. Jusqu'où cela s'est répandu est bien difficile à évaluer ${ }^{15}$.

Sans doute plus important est le fait que le Créationnisme Scientifique a engendré un climat et un contexte où la bataille pour combattre le système libéral scientifique s'est trouvé une légitimité. La Christian Coalition peut se rassurer, dans sa réflexion stratégique, qu'un espace conceptuel-politique a été dégagé pour la pensée fondamentaliste, participant à ce qu'on appelle le mouvement majoritaire. En ce sens, la théologie du déluge de Morris est moins importante en raison de ses idées particulières et de sa théologie que parce qu'elle sème le doute sur la vision progressiste établie. Elle fait sérieux, et, de mon point de vue, elle devrait être prise au sérieux. Elle a suscité plusieurs recherches complètes et poussées de la part des philosophes des sciences, dont Kitcher (1982).

Son potentiel politique et sa capacité de remplacer la démocratie par une théocratie littéraliste n'est plus une simple fantaisie. En ce sens, le Créationnisme Scientifique a réussi à ouvrir un espace de légitimation de la pensée fondamentaliste au sein de la population, et il semble étendre son influence.

Son importance intellectuelle tient à la confusion de notre époque. Elle refait surface, de mon point de vue, chaque fois qu'il y a crise de sens. À semblables époques (Buber, 1965), une diversité de personnes perdent le sens de leur vie, sont dépouillées de leur sens de l'orientation et de leur compétence personnelle. Le moment bouge trop vite, nous perdons notre identité. Peut-être cela tient-il beaucoup à la révolution technologique, en particulier la télé, aux communications, aux ordinateurs (Gergen, 1991) ou peut-être à ce que certains appellent la bureaucratisation de l'esprit.

Dans des temps comme ceux-là, bien des gens se tournent vers différentes sources d'aide: les anciens textes philosophiques ou religieux, le mysticisme, le nationalisme ou l'ethnocentrisme, la drogue et ainsi de

15 Lors des dernières élections présidentielles de 1992, le compte approximatif des chrétiens fondamentalistes, plus ou moins regroupés entre eux par la Christian Coalition de Pat Robertson, était estimé à environ $8 \%$ de la population américaine... et «ils votent tous», disent-ils. 
suite. Quoiqu'il en soit du post-modernisme, il rallie aussi - sans le vouloir ni le savoir selon mes observations - l'attaque fondamentaliste contre la science, contre le soi-disant projet des Lumières de l'objectivité scientifique, et même contre la possibilité de toute vérité transcendante. Comme Nietzsche l'avait prédit au siècle dernier, il y a eu une montée du nihilisme européen: un dérapage accentué du simple scepticisme au scepticisme à propos de la possibilité même de connaître, puis au cynisme et finalement au nihilisme. Pour bien des personnes, cela contribue moins à la critique qu'à la destruction dans leur propre quête d'identité, distrayant de la critique sociale et ouvrant un espace propice à un retour des diverses formes de spiritualité, y compris le fondamentalisme.

Le Créationnisme Scientifique est une réponse à ce sentiment de destruction du sens et d'érosion de l'identité. Comme les autres retours à la compétence des textes, il promet la vérité et la sécurité de la connaissance absolue. Dans le cas du Créationnisme Scientifique, il semble promettre au moins une sorte de vérité et de compétence tout aussi bonne et certaine que celle de la science. À toute époque de grand doute et de dépression économique, sa théo-politique peut s'avérer puissante.

\section{Bibliographie}

AUGUSTINE, Saint. 1958. On Christian Doctrine. Tr. D.W. Robertson Jr., Indianapolis, Bobbs-Merrill.

BUBER, Martin. 1965. Between man and man. New York, Macmillan.

BUNYAN, John. 1987. Pilgrim's Progress. London, Wordworth.

CHRISTOPHER, Robert C. 1989. Crashing the Gates: the de WASPing of America's Power Elite. New York/Toronto, Simon \& Schuster.

CONWAY, F. \& J. SIEGELMAN. 1978. Snapping: America's Epidemic of Sudden Personality Change. New York, Dell Publ. Co.

FELDMAN, David M. 1968. Marital Relations, Birth Control, and Abortion in Jewish Law. New York, Schocken Books.

GERGEN, Kenneth J. 1991. The Saturated Self: Dilemmas of Identity in Contemporary Life. New York, Basic Books.

HEARNE, Vicki. 1986. Adam's Task: calling Animals by Name. New York, William Heinemann.

HOAGLAND, Hudson. 1964. «Science and the New Humanism», Science 143 (jan.10.): 111. 
HUNTER, James D. 1991. Culture Wars: the Struggle to Define America. New York, Basic Books.

KITCHER, Philip. 1982. Abusing Science: the Case Against Creationism. Cambridge, MIT Press.

LIPSET, S.M. 1969. «Prejudice and Politics in the American Past and Present», dans Prejudice, U.S.A. (GLOCK \& SIEGELMAN (ed.), New York, Praeger, 17-69.

MARTY, Martin E. 1981. "Believing in Adam \& Eve: the Surge in Religious Fundamentalism Reflects a Profound Yearning for a Return to Simple Values», Prime Time (aug. 1981): 21-26.

METZGER, Walter P. 1955. Academic Freedom in the Age of the University. New York, Columbia University Press.

MORRIS, Henry M. (ed). 1974. Scientific Creationism. San Diego, Creation-Life Publishers.

MULLER, H.J. 1958. «Human Values in Relation to Evolution», Science 127 (march. 21): 629.

NELKIN, Dorothy. 1977. Science Textbook Controversies and the Politics of Equal Time. Cambridge, MIT Press.

SARLES, Harvey B. 1985. Language and Human Nature. Minneapolis, University of Minnesota Press.

IDEM. $1993 \mathrm{~ms}$. The Foundations Project. (Cet ouvrage comprend un certain nombre d'études explorant les thèmes tels la crise du sens, la nature humaine, l'origine du sens, l'identité, etc.).

IDEM. $1993 \mathrm{~ms} 2$. A Religious Point of View. (Essai visant à tenter de caractériser le mode de pensée fondamentaliste).

WILLS, Garry. 1990. Under God: Religion and American Politics. New York/Toronto, Simon \& Schuster. 